\title{
Nanostructured Thin Coatings from Chitosan and an Elastin-Like Recombinamer with Acute Stimuli-Responsive Behavior
}

\author{
Rui R. Costa ${ }^{1,2, a}$, Artur J. Ribeiro ${ }^{3,4, b}$, José C. Rodríguez-Cabello $3,4, c$ \\ and João F. Mano ${ }^{1,2, d}$ \\ 13B's Research Group - Biomaterials, Biodegradables and Biomimetics, University of Minho, \\ AvePark, Zona Industrial da Gandra, S. Cláudio do Barco, 4806-909 Caldas das Taipas - \\ Guimarães, Portugal. \\ ${ }^{2}$ ICVS/3B's - PT Government Associate Laboratory, Braga/Guimarães, Portugal. \\ ${ }^{3}$ G. I. R. Bioforge, University of Valladolid, Edificio I+D, Paseo de Belén, 1, 47011, Valladolid, \\ Spain. \\ ${ }^{4}$ Networking Research Center on Bioengineering, Biomaterials and Nanomedicine (CIBER-BBN), \\ Valladolid, Spain. \\ arui.costa@dep.uminho.pt, barturjamr@gmail.com, 'cabello@bioforge.uva.es, \\ ${ }^{d j m a n o @ d e p . u m i n h o . p t ~}$
}

Keywords: Surface engineering; Layer-by-layer; Biomaterials; Recombinant polymers.

\begin{abstract}
In the present work, chitosan (CHI) and elastin-like recombinamers (ELRs) were used to conceive nanostructured thin films driven by sequential electrostatic layer-by-layer (LbL), a simple and versatile technique that discards the use of harmful reagents. Two similar ELRs were engineered to contain negatively charged aminoacids and organized and a single monoblock or a triblock. The buildup of the films was monitored in real time using a quartz-crystal microbalance with dissipation monitoring (QCM-D). Wettability transitions were observed from a moderate hydrophobic surface to an extremely wettable upon increasing the temperature to $50{ }^{\circ} \mathrm{C}$, accompanied by topography changes at the nanoscale as assessed by atomic force microscopy (AFM). Furthermore, the dependence on time for the surface molecular rearrangement was studied for the films with each ELR. The potential of this technology may stimulate the development of devices and biomaterials for biomedical applications in the near future, such as surfaces with tunable and patterned cell adhesion, while the use of ELRs will allow developing polypeptides with biological significance.
\end{abstract}

\section{Introduction}

The modification of surfaces has been a key aspect in biology and biotechnology, including cell expansion, development of biomaterials and preparation of substrates for regenerative medicine [13]. The surface is the first contact with the organism and dictates the subsequent biological events, namely the cellular events and biointegration. This is an approach with potential impact not only on the properties of current healthcare systems but also in more cutting-edge ones in tissue engineering.

Although several techniques may be used to modify a surface, the last decade has emphasized non-harmful and versatile techniques to modify polymeric substrates: the sequential adsorption of proteins and polysaccharides is one of the most promising today. This approach is known as layerby-layer and is driven by a multitude of intermolecular interactions - such as electrostatic contacts, hydrophobic interactions, and hydrogen bonding - that may occur between two distinct materials [4$6]$.

Much work has been done in multilayered systems using poly(styrene sulfonate)/poly(allylamine hydrochloride) and $\mathrm{CHI} /$ hyaluronan [7]. However, the knowledge obtained from such systems is not always easily extrapolated to other materials and that limits the use of potentially more relevant combinations for LbL in biomedical applications. In this work, we aimed to apply the concept of 
LbL to CHI used along with ELRs to conceive multilayered substrates driven by electrostatic interactions. While chitosan is a well-known biocompatible polycation of natural origin [8,9], ELRs are genetically engineered polypeptides mimicking the sequence of natural elastin, showing smart behavior towards external stimuli, among them temperature and $\mathrm{pH}$ [10]. We hypothesized that different ELRs, although similar in nature and sequence, would display distinct adsorption kinetics. Therefore, we biosynthesized a negatively charged ELR organized as a single block, and an ELR organized into three blocks - two containing the negative residues and one nonpolar - henceforth designated monoblock and triblock, respectively. Furthermore, we studied the stimuli-responsive properties of the assembled coatings in terms of wettability and topography variations.

\section{Materials and methods}

ELRs Expression and Purification. Gene expression of recombinant Escherichia coli strains were induced in a $12 \mathrm{~L}$ Applikon fermenter under $37{ }^{\circ} \mathrm{C}$ and controlled $\mathrm{pH}$ (7.00). Subsequent to fermentation, the culture was harvested and lysed by ultrasonic disruption. Insoluble remains were removed by centrifugation and the cleared lysate was subjected to purification based on an temperature cycles. The polymer in solution was then freeze-dried.

Buildup Kinetics Monitoring. An E4 QCM-D, from Q-Sense (Sweeden) was used to monitor the adsorption of $\mathrm{CHI}$ and the ELRs. Aqueous solutions of each polymer were prepared at concentrations of $100 \mu \mathrm{g} \cdot \mathrm{mL}^{-1}, \mathrm{pH} 4.5$ and in $0.15 \mathrm{M} \mathrm{NaCl}$. The combinations of $\mathrm{CHI} / \mathrm{monoblock}$ and $\mathrm{CHI} /$ triblock and their depositions were studied in parallel at the surface of gold-coated sensors, at $25^{\circ} \mathrm{C}$. The adsorption time was 10 minutes.

Contact Angle Measurements. Contact angles were measured in a DataPhysics OCA15 system using a phosphate-buffer saline (PBS) solution for sessile drop experiments. Coatings of $\mathrm{CHI} /$ monoblock and $\mathrm{CHI} /$ triblock assembled onto glass $\left(1 \mathrm{~cm}^{2}\right)$ were tested for their wettability at 4 and $50{ }^{\circ} \mathrm{C}$. The number of layers was defined based on the results from the QCM-D. The coatings were immersed in PBS placed under each temperature condition. After 10 minutes, the samples were withdrawn and contact angles were measured.

Topography Studies. AFM tests were conducted in a MultiMode STM microscope controlled by a NanoScope III from Digital Instruments system, operating in tapping mode at a frequency of $1 \mathrm{~Hz}$. A similar protocol to that of contact angle measurements was adopted. To assess the influence of $\mathrm{pH}$ in the coatings, a similar protocol to the contact angle measurements was followed. The samples were immersed in PBS at room temperature and $50{ }^{\circ} \mathrm{C}$ and then retrieved and introduced in the device. $\mathrm{pH}$ was also analyzed, for values of 7.4 and 12 . The analyzed area was $1 \mu \mathrm{m}^{2}$.

\section{Results and Discussion}

ELRs Peptide Sequence. Under the scope of electrostatic LbL, we developed two ELRs containing negative aminoacids - namely aspartic acids (D) and glutamic acids (E) - in order to bond with the protonated amine groups of CHI, positively charged under acidic conditions. Other aminoacids are present, however, like lysines $(\mathrm{K})$, although in lower number. Their final sequence is justified by the fact that these recombinamers include motifs with potential for biomineralization, which may prove to be useful for future bone regeneration applications. The representative structures of the monoblock and triblock are shown in Fig. 1, along with the corresponding aminoacidic sequence.

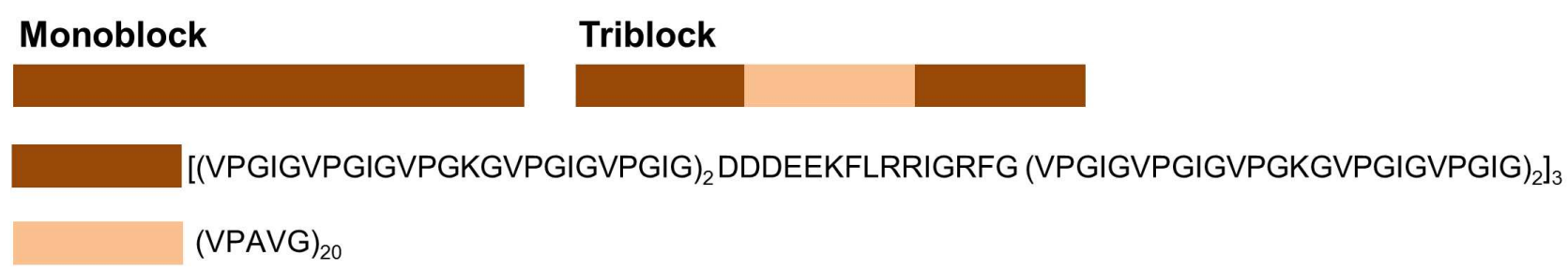

Fig. 1. Representative structure of the featured ELRs and corresponding block peptide sequence. 
The genetic nature of ELRs ultimately allows constructing macromolecules that are more appropriate for coatings of medical devices, allowing even to include sequences of biological relevance. However, their use for such purpose falls short of expectation and few works exist that use ELRs as ingredients for thin films [11-13]. Comparing both recombinamers, the triblock is different from the monoblock as it contains a polypeptide based on the pentapeptide VPAVG in the middle of the structure. This variation was introduced to evaluate if the buildup kinetics changes from the use of one ELR to another along with CHI for LbL, although their peptide sequence is similar, and to predict if other types of modifications would have the potential to be more favorable for multilayered buildup.

Buildup Monitoring of the Films. The ability of CHI and each ELR to adsorb in a sequential fashion was first monitored by QCM-D. This technique is able to detect the adsorption of molecules in the order of ng. $\mathrm{cm}^{-2}$ manifested by the decrease of the oscillation frequency of the sensor. It can also measure the viscoelastic properties of the resulting surface [14]. Fig. 2 shows the frequency and dissipation variations $\left(5^{\text {th }}\right.$ overtone, $25 \mathrm{MHz}$ ) for $\mathrm{CHI} /$ monoblock and $\mathrm{CHI} /$ triblock combinations.
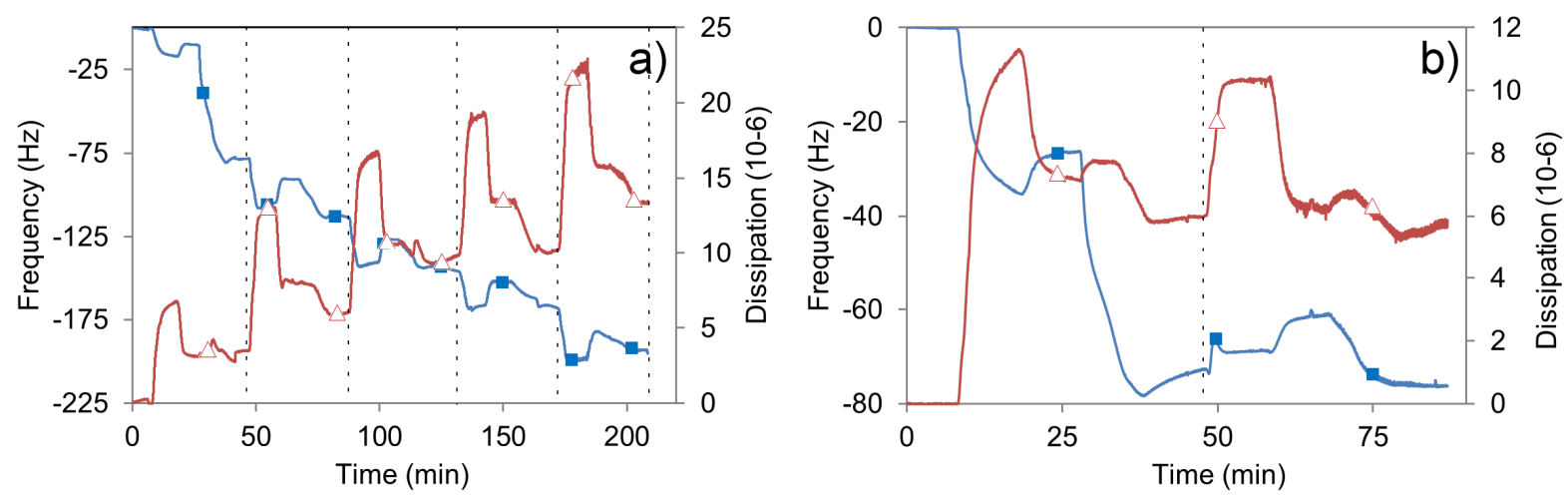

Fig. 2. QCM-D monitoring of the frequency ( $\mathbf{a})$ and dissipation $(\Delta)$ variations for a) $\mathrm{CHI} /$ monoblock and b) $\mathrm{CHI} /$ triblock. The dashed lines mark the end of the adsorption of one bilayer.

The first case, depicted in Fig. 2a, shows a progressive decrease of frequency, $\Delta \mathrm{F}$, to an approximate value of $200 \mathrm{~Hz}$, upon flushing the sensors with the sequence of $\mathrm{CHI}$ and the monoblock, up to 5 bilayers. Simultaneously, the overall dissipation, $\Delta \mathrm{D}$, increases, showing that the film exhibits viscoelastic behavior, typical of macromolecular systems [14,15]. It is worth mentioning that the dissipation decreases during the adsorption steps of the monoblock, corresponding to an increase of rigidity of the film. This characteristic may be usefull for the conception of coatings where a more rigid film is advantageous, like for bone regeneration devices.

In Fig. $2 b$, the monitoring of the $\mathrm{CHI} /$ triblock buildup is shown. As observed, only 1 bilayer of these polyelectrolytes was successfully adsorbed, up to a $\Delta \mathrm{F}$ of aroud $80 \mathrm{~Hz}$. The $\Delta \mathrm{F}$ after the attempt to deposit a second bilayer displays approximately the same value, therefore the following $\mathrm{CHI}$ and triblock molecules did not adsorb to the interface. This behavior can be explaned by the organization of the ELR: since the negatively charged blocks bond with the polycationic CHI, the nonpolar one does not intervene in the electrostatic-driven buildup. Therefore it faces outwards the interface of the film, rendering it uncharged, limiting the buildup to a single bilayer. When the adsorption of CHI is attempted, it does not adsorb because of the absence of negative aminoacids from the ELR. The results from the QCM-D show that, although the selected ELRs are similar in nature and peptide sequence, the buildup kinetics along with $\mathrm{CHI}$ is different, as previously theorized.

Wettability Variations of the Films. ELRs have an intrinsic smart response towards temperature in aqueous solutions. Therefore we expected to observe some variation of the wettability of each film with the temperature. The study of the buildup kinetics of $\mathrm{CHI}$ along with a monoblocked or triblocked ELR was shown in the previous section. Based on the QCM-D data, we tested coatings made of 5 bilayers of $\mathrm{CHI}$ and the monoblock $-(\mathrm{CHI} / \text { monoblock })_{5}-$ and 1 bilayer of $\mathrm{CHI}$ and the 
triblock - (CHI/triblock $)_{1}$. Contact angle measurements are easy and quick to perform and may give useful insight about the molecular arrangement of a polymer at a planar surface. It is particularly convenient when the wettability between two arrays of conditions is significantly different. Fig. 3 shows the contact angle variations caused by cyclic changes of the temperature - from $4{ }^{\circ} \mathrm{C}$ to $50{ }^{\circ} \mathrm{C}$ and vice-versa - and their evolution with time.

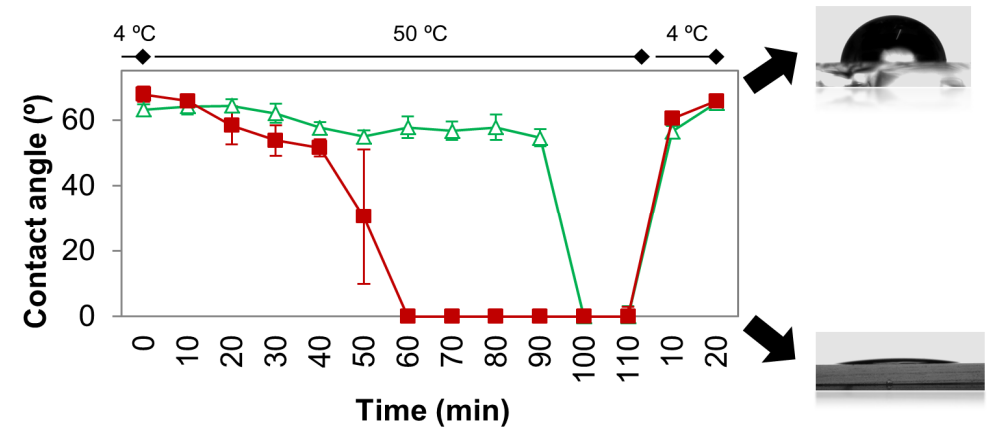

Fig. 3. Contact angle variations of $(\mathrm{CHI} / \text { monoblock })_{5}(\mathbf{\square})$ and $(\mathrm{CHI} / \text { triblock })_{1}(\Delta)$ upon temperature changes and evolution with time.

At start, the samples were at equilibrium at $4{ }^{\circ} \mathrm{C}$ and displayed a moderately hydrophobic feature, around $65^{\circ}$. Upon increasing the temperature to $50^{\circ} \mathrm{C}$, a transition was detected to a completely wettable surface in both cases. We have previously demonstrated that this observation is the result of the ELR transition at the surface due to the triggering of its self-assemble [12]. As a result of such phenomenon, there is a molecular rearrangement that favors the exposure of charged residues to the aqueous surroundings, while burying the hydrophobic aminoacids underneath.

The transition time was different for each sample, though. While in the case of $(\mathrm{CHI} / \text { monoblock })_{5}$ the superhydrophilic regimen was observed after 60 minutes, for the $(\mathrm{CHI} / \text { triblock })_{1}$ it took 100 minutes. This longer time can be explained by the exposure of the nonpolar alanines at the interface: the molecular rearrangement is more delayed, since at first the free charged aminoacids are present below the nonpolar block of the ELR, hindering the rearrangement. The transition is only later fully achieved. This does not happen with the monoblock, since its structure does not contain the highly hydrophobic portion (see Fig. 1). Upon decreasing the temperature to $4{ }^{\circ} \mathrm{C}$, both films recovered the moderately hydrophobic feature within 20 minutes. This result reflects the stimuli-responsive behavior of the films due to the properties of ELRs in aqueous solutions. Such behavior is retained and is cyclic when in the form of bidimensional substrates.

Topography Analysis. We determined that the monoblocked ELR is appropriate for constructing multilayered films. Having in mind that we intend to use ELRs using a multilayer approach along with polysaccharides, we studied the topography of $\mathrm{CHI} /$ monoblock films with different number of layers. The wettability variations found in the previous section should be accompanied by topography changes. Fig. 4 shows the images obtained by AFM for 1,3 and 5 bilayers. The variations of the topography with temperature were also studied, for 25 and for $50{ }^{\circ} \mathrm{C}$. Table 1 shows the root-mean-square roughness (rms) values for each case. As observed, independently of the temperature, the roughness increases with increasing number of layers due to the increase of mass at the surface, a typical observation of several LbL systems[5].

Table 1. Root-mean-square roughness for $(\mathrm{CHI} / \text { monoblock })_{5}$ films for 1,3 and 5 bilayers, at 25 and $50^{\circ} \mathrm{C}$, or $\mathrm{pH} 7.4$ and $\mathrm{pH} 14$, respectively.

\begin{tabular}{cccc}
\hline & \multicolumn{3}{c}{ Roughness, rms [nm] } \\
\hline Temperature $\left[{ }^{\circ} \mathrm{C}\right]$ or $\mathrm{pH}$ & 1 bilayer & 3 bilayers & 5 bilayers \\
\hline 25 or 7.4 & 0.874 & 0.905 & 1.482 \\
50 or 14 & 2.652 & 2.833 & 3.679
\end{tabular}




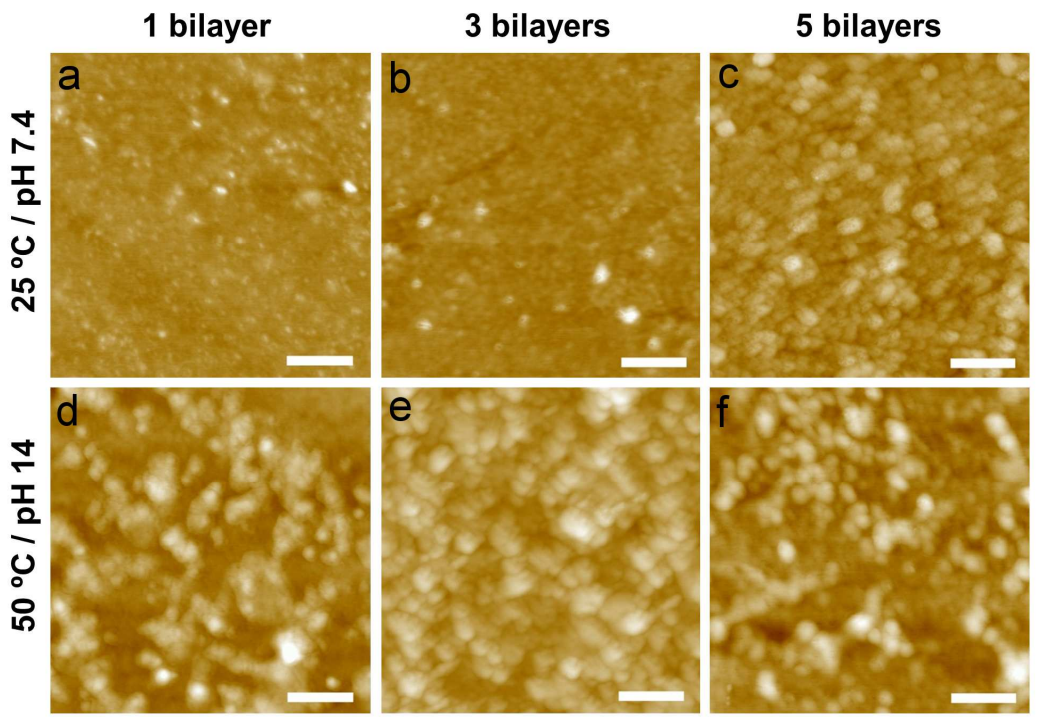

Fig. 4. AFM imaging for (CHI/monoblock $)_{5}$ films for 1,3 and 5 bilayers, at 25 and $50{ }^{\circ} \mathrm{C}$. Similar results were obtained for $\mathrm{pH} 7.4$ and $\mathrm{pH} 14$.

In Fig. 3a to Fig. 3c the topographies at room temperature show no apparent organization for any of the number of layers. Upon increasing the temperature to $50^{\circ} \mathrm{C}$, the formation of nanostructures was detected, caused by the triggering of the ELR self-assembly and consequent formation of larger and organized structures. The roughness values also increased in accordance. These results relate well with the contact angle measurements: the detected nanostructures result from the molecular rearrangement of the ELR, where their cores are mainly composed of nonpolar aminoacids, while exposing the charged residues to the outer regions. Therefore, these are micelle-like structures which organization favors the affinity of the films to water. It is also worth mentioning that similar topography and roughness results were obtained for the variation of $\mathrm{pH}$, which is also known to trigger the transition of ELRs containing lysines ( $\mathrm{pk}_{\mathrm{a}}$ ranging 9.4-10.6 [16]). Such organization at the nanoscale may be used in the future development of patterned substrates for tunable cell adhesion.

\section{Conclusions}

The presented work shows that polysaccharides and ELRS can be successfully combined following a multilayer approach to conceive nanostructured films. Both ELRs are similar in nature and peptide sequence; however the buildup kinetics is different, being limited to only 1 bilayer in the case of the triblock. Acute wettability variations were observed as a function of temperature for coatings of $\left(\mathrm{CHI} / \mathrm{monoblock}_{5}\right.$ and $(\mathrm{CHI} / \text { triblock })_{1}$, caused by a molecular rearrangement of the ELRs at the surface. Topography changes were detected at the nanoscale, where micelle-like structures were formed due to the increase of temperature or $\mathrm{pH}$. The properties of the films may be exploited in the future for substrates with patterned and tunable cell adhesion. Furthermore, the recombinant nature of such elastin-like macromolecules may enable tuning their sequence to favor LbL constructs and even adding biological relevant motifs.

\section{Acknowledgements}

The authors acknowledge the financial support through Fundação para a Ciência e Tecnologia (FCT, PhD grant SFRH/BD/61126/2009, “Junta de Castilla y Leon'” (VA034A09), the MICINN (MAT 2009-14195-C03-03, ACI2009-0890, IT2009-0089, MAT2010-15310, and MAT201015982), the CIBER-BBN and the "Network Center of Regenerative Medicine and Cellular Therapy of Castilla y León'” 


\section{References}

[1] D. Falconnet, G. Csucs, H. Michelle Grandin, M. Textor, Surface engineering approaches to micropattern surfaces for cell-based assays, Biomaterials, 27 (2006) 3044-3063.

[2] A.L. Hook, N.H. Voelcker, H. Thissen, Patterned and switchable surfaces for biomolecular manipulation, Acta Biomaterialia, 5 (2009) 2350-2370.

[3] M. Tirrell, E. Kokkoli, M. Biesalski, The role of surface science in bioengineered materials, Surface Science, 500 (2002) 61-83.

[4] N.M. Alves, I. Pashkuleva, R.L. Reis, J.F. Mano, Controlling cell behavior through the design of polymer surfaces, Small, 6 (2010) 2208-2220.

[5] P. Bertrand, A. Jonas, A. Laschewsky, R. Legras, Ultrathin polymer coatings by complexation of polyelectrolytes at interfaces: suitable materials, structure and properties, Macromolecular Rapid Communications, 21 (2000) 319-348.

[6] G. Decher, Fuzzy Nanoassemblies: Toward layered polymeric multicomposites, Science, 277 (1997) 1232-1237.

[7] T. Boudou, T. Crouzier, K. Ren, G. Blin, C. Picart, Multiple functionalities of polyelectrolyte multilayer films: new biomedical applications, Advanced Materials, 22 (2010) 441-467.

[8] N.M. Alves, J.F. Mano, Chitosan derivatives obtained by chemical modifications for biomedical and environmental applications, International Journal of Biological Macromolecules, 43 (2008) 401-414.

[9] M.N.V. Ravi Kumar, A review of chitin and chitosan applications, Reactive and Functional Polymers, 46 (2000) 1-27.

[10] J.C. Rodríguez-Cabello, L. Martín, M. Alonso, F.J. Arias, A.M. Testera, "Recombinamers" as advanced materials for the post-oil age, Polymer, 50 (2009) 5159-5169.

[11] J. Barbosa, R. Costa, A. Testera, M. Alonso, J. Rodríguez-Cabello, J. Mano, Multi-layered films containing a biomimetic stimuli-responsive recombinant protein, Nanoscale Research Letters, 4 (2009) 1247-1253.

[12] R.R. Costa, C.A. Custódio, A.M. Testera, F.J. Arias, J.C. Rodríguez-Cabello, N.M. Alves, J.F. Mano, Stimuli-responsive thin coatings using elastin-like polymers for biomedical applications, Advanced Functional Materials, 19 (2009) 3210-3218.

[13]M. Swierczewska, C.S. Hajicharalambous, A.V. Janorkar, Z. Megeed, M.L. Yarmush, P. Rajagopalan, Cellular response to nanoscale elastin-like polypeptide polyelectrolyte multilayers, Acta Biomaterialia, 4 (2008) 827-837.

[14]K.A. Marx, Quartz crystal microbalance: a useful tool for studying thin polymer films and complex biomolecular systems at the solution-surface interface, Biomacromolecules, 4 (2003) 1099-1120.

[15]C. Picart, P. Lavalle, P. Hubert, F.J.G. Cuisinier, G. Decher, P. Schaaf, J.C. Voegel, Buildup mechanism for poly(L-lysine)/hyaluronic acid films onto a solid surface, Langmuir, 17 (2001) 74147424.

[16]L.A. Fothergill-Gilmore, Peptide sequence determination, in: F. Franks (Ed.), Protein biotechnology: isolation, characterization, and stabilization, Humana Press, Totowa NJ, 1993, pp. 254-256. 
Advanced Materials Forum VI

10.4028/www.scientific.net/MSF.730-732

Nanostructured Thin Coatings from Chitosan and an Elastin-Like Recombinamer with Acute Stimuli-Responsive Behavior

10.4028/www.scientific.net/MSF.730-732.32 